\title{
Analysis of the sex-specific variability of blood parameters in data sets of the Mouse Phenome Database
}

\author{
Bernhard Aigner* (10
}

\begin{abstract}
Objective: The use of mice as animal models in biomedical research allows the standardization of genetic background and environmental conditions, which both affect phenotypic variability. As the use of both sexes in experiments is strongly recommended, sex-specific phenotypic variability is discussed with regard to putative consequences on the group size which is necessary for achieving valid and reproducible results. In this study, the sex-specific variability of 25 clinical chemical and hematological parameters which represent a comprehensive blood screen of laboratory mice, was analyzed in data sets which have been submitted to the Mouse Phenome Database.

Results: The overall analysis comprising all 25 clinical chemical and hematological parameters showed no evidence for substantial and robust general sex-specific variability. A large range of the ratio of the female and male coefficient of variation (CV) was found for every parameter among the respective strain data sets. This clearly demonstrated the appearance of unpredictable major interactions between genotype and environment regarding the sex-specific variability of the blood parameters analyzed.
\end{abstract}

Keywords: Animal model, Clinical chemistry, Hematology, Sex, Variability

\section{Introduction}

When carrying out biomedical research with animal models, the extent of the phenotypic variability affects the group size which has to be used in the experiment for achieving valid and reproducible results. The use of both sexes in the experiments is strongly recommended because of possible differences in the outcome $[1,2]$. Therefore, the appearance of sex-specific phenotypic variability is discussed in the context of differences in the social status especially in male group housed mice as well as of non-synchronous hormone cycles in female experimental groups which may alter the homogeneity of study

*Correspondence: b.aigner@gen.vetmed.uni-muenchen.de Chair for Molecular Animal Breeding and Biotechnology, and Laboratory for Functional Genome Analysis (LAFUGA), Gene Center, LMU Munich, 81377 Munich, Germany populations and subsequently confound effects of experimental manipulations ([3-6] and refs. therein).

Meta-analyses examined published mouse data concerning this topic $[4,5,7]$. Prendergast et al. examined behavioral, morphological, physiological, and molecular traits in 293 articles, which were monitored in male mice and females tested without regard to the estrous cycle stage. The variability was not significantly greater in females than males for any endpoint and was substantially greater in males for several traits. In addition, group housing of mice was observed to increase the variability in both males and females by $37 \%$ [4].

In this study, the sex-specific variability of 25 clinical chemical and hematological parameters of laboratory mouse strains was analyzed in data sets which have been submitted to the Mouse Phenome Database. The blood parameters represent a comprehensive blood screen and 
were chosen as quantitative parameters for the analysis because they reflect a large range of phenotype traits.

\section{Main text \\ Methods}

The following ontology terms (VT, vertebrate trait ontologies) were used for the selection of the project data sets of the chosen parameters in the Mouse Phenome Database (https://phenome.jax.org): cholesterol, VT:0000180; creatinine, VT:0005328; glucose, VT:0000188; total protein, VT:0005567; triglycerides, VT:0002644; urea, VT:0005265; uric acid, VT:0010302; ferritin, VT:0010513; transferrin, VT:0010514; calcium, VT:0001562; chloride, VT:0003018; phosphorus, VT:0001565; potassium, VT:0002668; sodium, VT:0001776; alanine aminotransferase (ALT, EC 2.6.1.2), VT:0001573; aspartate aminotransferase (AST, EC 2.6.1.1), VT:0000203; $\alpha$-amylase (EC 3.2.1.1), VT:0010475; alkaline phosphatase (AP, EC 3.1.3.1), VT:0000202; creatine kinase (CK, EC 2.7.3.2), VT:1000047; lipase (EC 3.1.1.3), VT:0010478; hemoglobin, VT:0001588; mean corpuscular volume (MCV), no VT applied; red blood cell count (RBC), VT:0001586; white blood cell count (WBC), VT:0000217; platelets, VT:0003179. The parameters hematocrit, mean corpuscular hemoglobin $(\mathrm{MCH})$, and mean corpuscular hemoglobin concentration $(\mathrm{MCHC})$ were not included in the study as they are subsequently calculated by using parameters which were directly measured.

Within each selected project data set, strain data sets were chosen for the analysis of the coefficient of variation $(\mathrm{CV}=$ standard deviation/mean) of males and females within the same data set by using following selection criteria: inbred strains [including those derived from the Collaborative Cross (CC)], F1 hybrids, recombinant inbred strains; no lines with newly generated alleles; no treatment; age of the mice examined: 7-26 weeks; group size: $\mathrm{n} \geq 5$ for each sex; as of December 2020 .

Strain data sets with unusually high phenotypic variability for a given parameter due to e.g. technical outliers or unrecognized reasons-which was defined by the appearance of $\mathrm{CV}$ values $>0.5$ for the female and/or the male group-were excluded from the further analysis. This mainly occurs for the three parameters creatinine, ALT and CK. For all other parameters, 2.3\% (1.1\% female group outliers, $0.6 \%$ male group outliers, and $0.6 \%$ outliers in both the female and male groups) of the strain data sets were excluded as outliers. The additional analysis with all strain data sets included did not change the results described below. Data analysis was carried out using the software program Microsoft Excel 2016 (Microsoft Corp., Redmond, WA). The chi-squared test was used for the statistical analysis of the data.

\section{Results}

The phenotypic variability of 25 clinical chemical and hematological parameters was analyzed by determining the coefficient of variation ( $\mathrm{CV}=$ standard deviation/ mean) both for the male and female mice within a given strain data set. The extent of the $\mathrm{CV}$ value for both sexes depends on the phenotypic parameter analyzed (e.g. the red blood cell parameters hemoglobin, $\mathrm{MCV}$ and $\mathrm{RBC}$ as well as the electrolytes calcium, chloride and sodium usually exhibit relatively low $\mathrm{CV}$ values; and the enzyme activities ALT, AST and CK as well as the plasma substrate triglycerides exhibit relatively high $\mathrm{CV}$ values). Within a given strain data set, a CV ratio (=female $\mathrm{CV} /$ $($ female $\mathrm{CV}+$ male $\mathrm{CV}))<0.5$ indicates that the female $\mathrm{CV}$ is lower than the male $\mathrm{CV}$, whereas a $\mathrm{CV}$ ratio $>0.5$ indicates that the female $\mathrm{CV}$ is higher than the male CV.

The data analysis was first carried out by using all strain data sets where at least five mice were examined for each sex. For the 25 chosen blood parameters, the mean $\mathrm{CV}$ ratios ranged from 0.44 to 0.53 . Twelve parameters showed a mean $C V$ ratio $>0.5$, whereas the other twelve parameters showed a mean $\mathrm{CV}$ ratio $<0.5$ (no data sets were included for the parameter $\mathrm{CK}$ by the selection procedure described in "Methods" section). For a given parameter, the portion of strain data sets with a CV ratio $>0.5$ ranged from 24 to $75 \%$. The parameter $\mathrm{MCV}$ showed an inconsistency of the mean $\mathrm{CV}$ ratio vs. the portion of strain data sets showing a $\mathrm{CV}$ ratio $>0.5$ or $<0.5$, respectively (i.e. a mean $\mathrm{CV}$ ratio of 0.49 , whereas $55 \%$ of the strain data sets showed a $\mathrm{CV}$ ratio $>0.5$ ) (Table 1).

Subsequently, the data analysis was carried out by using only strain data sets where at least ten mice were examined for each sex. For the 25 chosen blood parameters, the mean $\mathrm{CV}$ ratios ranged from 0.39 to 0.53 . Nine parameters showed a mean $\mathrm{CV}$ ratio $>0.5$, whereas 14 parameters showed a mean $\mathrm{CV}$ ratio $<0.5$. A relatively high deviation from the hypothesized $\mathrm{CV}$ ratio of 0.5 (i.e. a $\mathrm{CV}$ ratio $<0.48$ or $>0.52$ ) was found for the parameters ferritin (mean $\mathrm{CV}$ ratio $=0.53$ of 4 data sets), transferrin (mean $\mathrm{CV}$ ratio $=0.46$ of 4 data sets), chloride (mean $\mathrm{CV}$ ratio $=0.46$ of 15 data sets), ALT (mean CV ratio $=0.39$ of 10 data sets), and $\mathrm{RBC}$ (mean $\mathrm{CV}$ ratio $=0.47$ of 111 data sets). Thus, except of the parameter RBC, in all other cases only a low number of strain data sets was available. For a given parameter, the portion of strain data sets with a CV ratio $>0.5$ ranged from 10 to $75 \%$. The parameters $\alpha$-amylase, MCV and platelets showed an inconsistency of the mean $\mathrm{CV}$ ratio vs. the portion of strain data sets showing a CV ratio $>0.5$ or $<0.5$, respectively (Table 1 ).

The comparison of the outcome of the data analyses " $n \geq 5 /$ sex" vs. " $n \geq 10 /$ sex" revealed inconsistent $C V$ ratios in both analyses, i.e. a $\mathrm{CV}$ ratio $>0.5$ in the analysis 


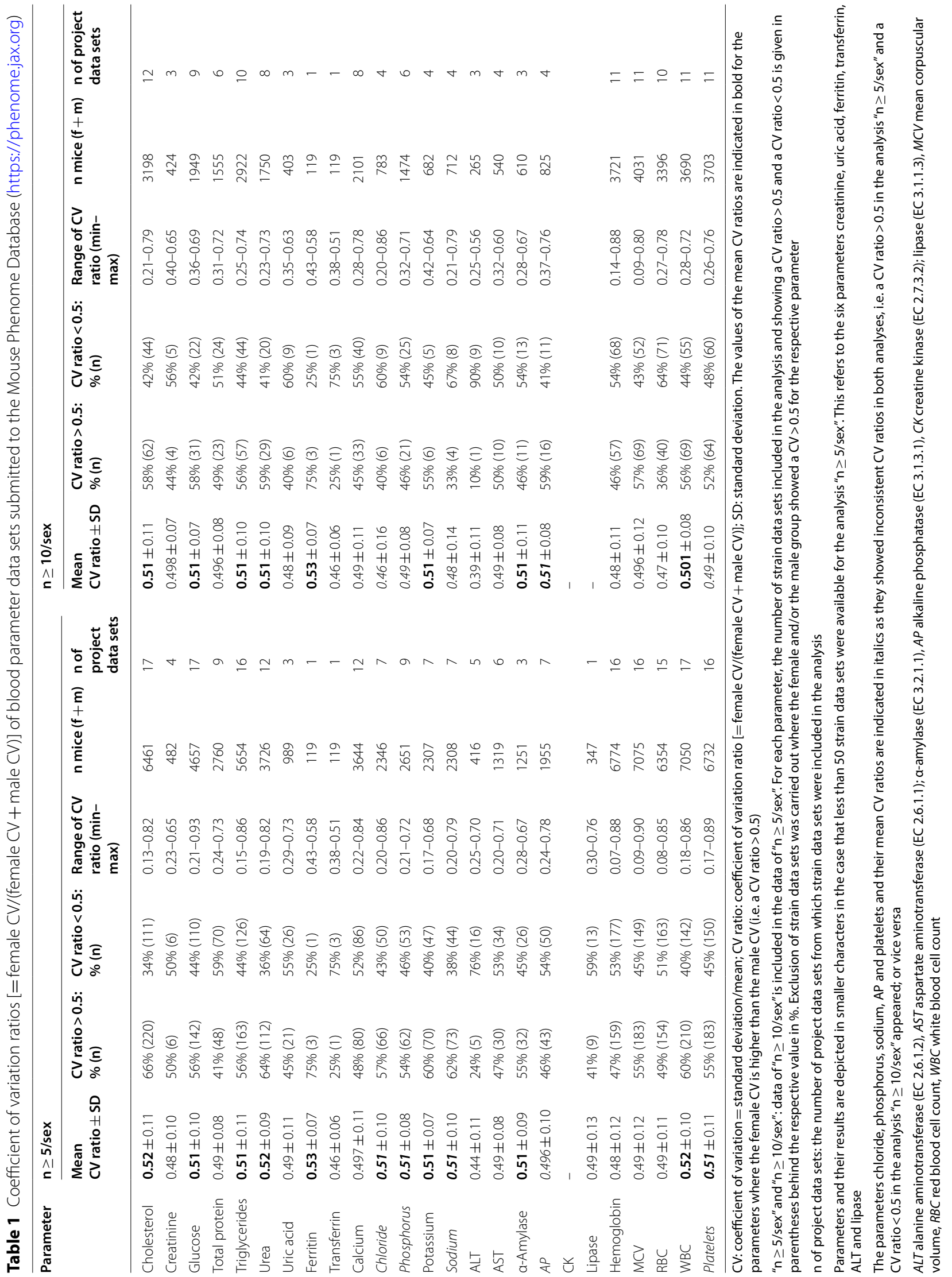


Table 2 Coefficient of variation ratios [= female CV/(female CV + male CV)] of blood parameter data sets of selected inbred strains submitted to the Mouse Phenome Database (https://phenome.jax.org)

\begin{tabular}{|c|c|c|c|c|c|c|}
\hline $\begin{array}{l}\text { Mean CV ratio } \pm S D \text { ( } n \text { of } \\
\text { data sets) }\end{array}$ & $A / J \ldots$ & BALB/c... & С $3 \mathrm{H} . .$. & C57BL/6... & $\mathrm{CBA} / \ldots$ & DBA/2J... \\
\hline Cholesterol & $\mathbf{0 . 5 6} \pm 0.09(9)$ & $\mathbf{0 . 5 4} \pm 0.05(13)$ & $\mathbf{0 . 5 3} \pm 0.10(10)$ & $\mathbf{0 . 5 1} \pm 0.13(16)$ & $\mathbf{0 . 5 1} \pm 0.16(8)$ & $\mathbf{0 . 6 1} \pm 0.12(7)$ \\
\hline Creatinine & $0.49 \pm 0.02(2)$ & $\mathbf{0 . 5 0} \pm 0.06(5)$ & $0.48 \pm 0.04$ & $\mathbf{0 . 5 1} \pm 0.08(6)$ & $\mathbf{0 . 5 3} \pm 0.06(3)$ & $0.50(1)$ \\
\hline Glucose & $0.60 \pm 0.10(7)$ & $0.45 \pm 0.12(11)$ & $\mathbf{0 . 5 2} \pm 0.05(8)$ & $\mathbf{0 . 5 2} \pm 0.09(17)$ & $0.48 \pm 0.06(6)$ & $\mathbf{0 . 5 9} \pm 0.10(5)$ \\
\hline Total protein & $0.49 \pm 0.04$ & $0.50 \pm 0.04(9)$ & $0.43 \pm 0.11(6)$ & $\mathbf{0 . 5 0} \pm 0.07(7)$ & $0.47 \pm 0.04$ & $\mathbf{0 . 5 5} \pm 0.07(4)$ \\
\hline Triglycerides & $\mathbf{0 . 5 5} \pm 0.10(9)$ & $0.50 \pm 0.14(13)$ & $\mathbf{0 . 5 4} \pm 0.07(10)$ & $\mathbf{0 . 5 2} \pm 0.16(16)$ & $\mathbf{0 . 5 5} \pm 0.10$ & $\mathbf{0 . 5 4} \pm 0.12(7)$ \\
\hline Urea & $\mathbf{0 . 5 0} \pm 0.18(5)$ & $0.45 \pm 0.11(11)$ & $\mathbf{0 . 5 6} \pm 0.09(8)$ & $\mathbf{0 . 5 2} \pm 0.12(10)$ & $\mathbf{0 . 5 4} \pm 0.10(6)$ & $\mathbf{0 . 5 2} \pm 0.15(5)$ \\
\hline Uric acid & $0.44(1)$ & $0.48 \pm 0.01(4)$ & $0.46(1)$ & $\mathbf{0 . 5 2} \pm 0.10(3)$ & $\mathbf{0 . 5 5} \pm 0.12(3)$ & $0.67(1)$ \\
\hline Ferritin & - & $0.58(1)$ & $0.53(1)$ & $0.58(1)$ & - & - \\
\hline Transferrin & - & $0.51(1)$ & $0.50(1)$ & $0.46(1)$ & - & - \\
\hline Calcium & $0.49 \pm 0.08(5)$ & $0.47 \pm 0.11(11)$ & $0.49 \pm 0.11(8)$ & $\mathbf{0 . 5 2} \pm 0.08(10)$ & $0.47 \pm 0.13(6)$ & $\mathbf{0 . 5 6} \pm 0.13(6)$ \\
\hline Chloride & $\mathbf{0 . 5 3} \pm 0.06(3)$ & $\mathbf{0 . 6 1} \pm 0.17(4)$ & $\mathbf{0 . 6 1} \pm 0.18(4)$ & $\mathbf{0 . 5 1} \pm 0.13(5)$ & $0.50(1)$ & $\mathbf{0 . 5 3} \pm 0.06(2)$ \\
\hline Phosphorus & $\mathbf{0 . 5 4} \pm 0.05(3)$ & $0.46 \pm 0.11(9)$ & $\mathbf{0 . 5 2} \pm 0.07(6)$ & $\mathbf{0 . 5 3} \pm 0.18(7)$ & $0.48 \pm 0.08(5)$ & $0.48 \pm 0.09(4)$ \\
\hline Potassium & $\mathbf{0 . 5 4} \pm 0.06(3)$ & $\mathbf{0 . 5 4} \pm 0.04(4)$ & $\mathbf{0 . 5 0} \pm 0.07(4)$ & $0.46 \pm 0.04(5)$ & $0.53(1)$ & $0.47 \pm 0.03(2)$ \\
\hline Sodium & $0.48 \pm 0.01(3)$ & $\mathbf{0 . 5 5} \pm 0.05(4)$ & $\mathbf{0 . 6 4} \pm 0.15(4)$ & $\mathbf{0 . 5 2} \pm 0.10(5)$ & $0.51(1)$ & $0.46 \pm 0.04(2)$ \\
\hline ALT & $0.47(1)$ & $0.46 \pm 0.08(4)$ & $0.41 \pm 0.04$ & $0.45 \pm 0.09(5)$ & $0.40 \pm 0.22(2)$ & $0.40 \pm 0.11(2)$ \\
\hline AST & $0.45 \pm 0.21(3)$ & $\mathbf{0 . 5 4} \pm 0.04(4)$ & $0.46 \pm 0.01(2)$ & $0.42 \pm 0.09(5)$ & $\mathbf{0 . 5 1} \pm 0.01(3)$ & $0.47(1)$ \\
\hline a-Amylase & $\mathbf{0 . 5 5} \pm 0.05(2)$ & $\mathbf{0 . 5 4} \pm 0.09(4)$ & $\mathbf{0 . 5 3} \pm 0.06(2)$ & $0.50 \pm 0.10(4)$ & $\mathbf{0 . 5 7} \pm 0.02(3)$ & $0.34(1)$ \\
\hline $\mathrm{AP}$ & $\mathbf{0 . 5 0} \pm 0.04(3)$ & $\mathbf{0 . 5 6} \pm 0.10(5)$ & $\mathbf{0 . 5 7} \pm 0.19(3)$ & $\mathbf{0 . 5 3} \pm 0.16(5)$ & $0.44 \pm 0.00(3)$ & $\mathbf{0 . 5 3} \pm 0.07(2)$ \\
\hline CK & - & $\mathbf{0 . 5 2} \pm 0.05(4)$ & $0.43 \pm 0.10(3)$ & $\mathbf{0 . 5 2} \pm 0.14(3)$ & $0.42 \pm 0.22(2)$ & $0.39 \pm 0.08(2)$ \\
\hline Lipase & $0.44(1)$ & $0.58(1)$ & $0.40(1)$ & - & - & $0.43(1)$ \\
\hline Hemoglobin & $\mathbf{0 . 5 1} \pm 0.17(10)$ & $0.47 \pm 0.12(15)$ & $0.46 \pm 0.07(10)$ & $0.45 \pm 0.08(15)$ & $0.50 \pm 0.07(9)$ & $0.45 \pm 0.08(8)$ \\
\hline MCV & $\mathbf{0 . 5 6} \pm 0.10(10)$ & $\mathbf{0 . 5 1} \pm 0.14(15)$ & $0.49 \pm 0.14(10)$ & $0.46 \pm 0.08(15)$ & $\mathbf{0 . 5 1} \pm 0.12(11)$ & $0.45 \pm 0.13(8)$ \\
\hline $\mathrm{RBC}$ & $\mathbf{0 . 5 2} \pm 0.12(9)$ & $0.45 \pm 0.12(14)$ & $0.49 \pm 0.06(9)$ & $0.48 \pm 0.09(14)$ & $\mathbf{0 . 5 2} \pm 0.09(10)$ & $0.39 \pm 0.11(7)$ \\
\hline WBC & $\mathbf{0 . 5 1} \pm 0.11(11)$ & $0.48 \pm 0.11(16)$ & $0.45 \pm 0.08(11)$ & $0.50 \pm 0.10(16)$ & $\mathbf{0 . 5 3} \pm 0.05(11)$ & $\mathbf{0 . 5 0} \pm 0.07(9)$ \\
\hline Platelets & $\mathbf{0 . 5 6} \pm 0.14(10)$ & $0.47 \pm 0.13(15)$ & $\mathbf{0 . 5 1} \pm 0.11(10)$ & $0.49 \pm 0.11(15)$ & $0.49 \pm 0.10(10)$ & $0.47 \pm 0.07(8)$ \\
\hline "n $\geq 5$ 5": n CV ratio > 0.5 & 9 of 10 & 4 of 14 & 6 of 12 & 11 of 19 & 6 of 12 & 6 of 10 \\
\hline "n $\geq 1 ":$ n CV ratio > 0.5 & 14 of 22 & 13 of 25 & 12 of 25 & 14 of 24 & 13 of 22 & 10 of 23 \\
\hline
\end{tabular}

$\mathrm{CV}$ : coefficient of variation = standard deviation/mean; $\mathrm{CV}$ ratio: coefficient of variation ratio $[=$ female $\mathrm{CV} /(\mathrm{female} \mathrm{CV}+$ male $\mathrm{CV})]$; SD: standard deviation. The values of the mean $\mathrm{CV}$ ratios are indicated in bold for the parameters where the female $\mathrm{CV}$ is higher than the male $\mathrm{CV}$ (i.e. a CV ratio >0.5). No exclusion of strain data sets was carried out in the case that the female and/or the male group showed a CV $>0.5$ for the respective parameter

Data sets with the designation $\mathrm{A} / \mathrm{J} . ., \mathrm{BALB} / \mathrm{c} \ldots, \mathrm{C} 3 \mathrm{H} \ldots, \mathrm{C} 57 \mathrm{BL} / 6 \ldots, \mathrm{CBA} / \ldots$ and $\mathrm{DBA} / 2 \mathrm{~J} \ldots$ were summarized for the respective inbred strains $\mathrm{A} / \mathrm{J}, \mathrm{BALB} / \mathrm{c}, \mathrm{C} 3 \mathrm{H}$, C57BL/6, CBA and DBA/2 J

" $n \geq 5$ " and " $n \geq 1$ ": the number of data sets available for a given parameter of a selected inbred strain is shown in parentheses behind the value of the mean CV ratio \pm SD. Results including less than five data sets are depicted in smaller characters. The analysis " $n \geq 5$ " exhibits the number of parameters with a CV ratio $>0.5$ compared to the number of all parameters with at least five data sets. The analysis " $n \geq 1$ " exhibits the number of parameters with a CV ratio $>0.5$ compared to the number of all parameters with at least one data set

$A L T$ alanine aminotransferase (EC 2.6.1.2), AST aspartate aminotransferase (EC 2.6.1.1); a-amylase (EC 3.2.1.1), $A P$ alkaline phosphatase (EC 3.1.3.1), CK creatine kinase (EC 2.7.3.2); lipase (EC 3.1.1.3), MCV mean corpuscular volume, $R B C$ red blood cell count, WBC white blood cell count

" $\mathrm{n} \geq 5 / \mathrm{sex}$ " and a CV ratio $<0.5$ in the analysis " $\mathrm{n} \geq 10$ / sex" or vice versa for the five parameters chloride, phosphorus, sodium, AP and platelets. Compared to the hypothesis of equal numbers of strain data sets with a $\mathrm{CV}$ ratio $<0.5$ and a $\mathrm{CV}$ ratio $>0.5$, the statistical examination of the detected counts of strain data sets with a $\mathrm{CV}$ ratio $>0.5$ and with a $\mathrm{CV}$ ratio $<0.5$ was carried out by using the chi-squared test to detect a consistent significant difference $(p<0.05)$ in both analyses " $n \geq 5 /$ sex" and " $n \geq 10 /$ sex" for a given parameter. This was only found for the parameter ALT; a parameter with only a low number of strain data sets available. In addition, large ranges (minimum-maximum) of the $\mathrm{CV}$ ratio appeared for more or less all parameters in both analyses " $n \geq 5 /$ sex" and " $\mathrm{n} \geq 10 /$ sex" (Table 1 ).

Subsequently, the data sets were analyzed with respect to the particular inbred strains which were identified as the most often examined strains for the 25 blood parameters. Again, only data sets with at least five mice for each sex examined were included. Here, strain data sets were 
not excluded in the case that the female and/or the male group shows a $\mathrm{CV}>0.5$ for a given parameter. For each of the six inbred strains $\mathrm{A} / \mathrm{J}, \mathrm{BALB} / \mathrm{c}, \mathrm{C} 3 \mathrm{H}, \mathrm{C} 57 \mathrm{BL} / 6, \mathrm{CBA}$ and $\mathrm{DBA} / 2 \mathrm{~J}$, ten or more parameters were achieved with results from five or more different project data sets. The numbers of parameters showing a mean $\mathrm{CV}$ ratio $>0.5$ were as follows for the particular inbred strains: 9 out of 10 for $\mathrm{A} / \mathrm{J}, 4$ out of 14 for BALB/c, 6 out of 12 for $\mathrm{C} 3 \mathrm{H}$, 11 out of 19 for C57BL/6, 6 out of 12 for CBA, and 6 out of 10 for DBA/2J (Table 2). Due to the low number of parameters with at least five data sets included in the analysis, the results may only provide hints for the potential appearance of sex-specific variability in particular blood parameters and/or specific strains.

\section{Discussion}

The Mouse Phenome Database (https://phenome.jax.org) provides reference values of a high number of phenotype parameters mostly for the inbred strains which are predominantly used in biomedical research. Therefore, the data sets from this database were chosen for the analysis of sex-specific variability. The overall analysis comprising all 25 clinical chemical and hematological parameters showed no evidence for a substantial general sex-specific variability.

It is assumed that the data sets have been achieved by using the housing method which is usually carried out when working with mice in biomedical research, i.e. both sexes are group housed, and females are used without regard to the stage of the estrous cycle. The group sizes of the strain data sets selected for this study may fit to the relatively low numbers of animals which are usually used as group size at least in fundamental biomedical research. A meta-analysis revealed that group housing of mice increased the variability in both males and females by $37 \%$ [4]. Therefore, no sex is expected to take advantage of this housing method in respect to the extent of the variability compared to the other sex. The similar increase of the variability in group housed males and females is also expected to cover the consequences of the Lee Boot effect which leads to the suppression of the estrous cycle in group housed female mice [8].

The analysis of the sex-specific variability of 25 blood parameters resulted in a relatively high deviation from the hypothesized $\mathrm{CV}$ ratio of 0.5 (i.e. a $\mathrm{CV}$ ratio $<0.48$ or $>0.52$ ) only for the parameter $\mathrm{RBC}$ (mean $\mathrm{CV}$ ratio $=0.47$ of 111 data sets in the analysis " $n \geq 10 /$ sex") where a sufficiently high number of strain data sets was available. In addition, a large range (minimum-maximum) of the $\mathrm{CV}$ ratio was found for every parameter among the respective strain data sets. This clearly demonstrated the appearance of unpredictable major interactions between genotype and environment regarding the sex-specific variability of the blood parameters analyzed. As no particular parameter is described with a robust difference in the phenotypic variability between both sexes which may be used as a "positive control", the published increase of the phenotypic variability $(=\mathrm{CV})$ within each sex by $37 \%$ in group housed animals [4] may be used to evaluate the results detected in this study. The difference of $37 \%$ would result in a CV ratio of 0.39 or 0.61 when comparing single housed mice and group housed mice. This deviation from the hypothesized CV ratio of 0.5 is much higher than the deviations of the mean $\mathrm{CV}$ ratios from the hypothesized $\mathrm{CV}$ ratio of 0.5 detected in our study for all parameters analyzed where a sufficiently high number of strain data sets was available.

\section{Limitations}

Data sets of the Mouse Phenome Database (https:// phenome.jax.org) provide reference values for the inbred strains predominantly used. It is assumed that the projects providing the strain data sets have been carried out by using standardized protocols, but not especially for the analysis of sex-specific phenotypic variability.

\section{Abbreviations}

ALT: Alanine aminotransferase; AP: Alkaline phosphatase; AST: Aspartate aminotransferase; CC: Collaborative Cross; CK: Creatine kinase; CV: Coefficient of variation; MCH: Mean corpuscular hemoglobin; MCHC: Mean corpuscular hemoglobin concentration; MCV: Mean corpuscular volume; RBC: Red blood cell count; VT:Vertebrate trait ontologies; WBC: White blood cell count.

\section{Acknowledgements}

Not applicable.

\section{Authors' contributions}

BA conceived and designed the experiment, carried out the data analysis, wrote the paper. The author read and approved the final manuscript.

\section{Funding}

Open Access funding enabled and organized by Projekt DEAL. No funding was received for the analysis.

\section{Availability of data and materials}

The data sets were downloaded from the Mouse Phenome Database (https:// phenome.jax.org) and selected as described in "Methods" section. The accession numbers that refer to the specific datasets used in this study are given as ontology terms (VT, vertebrate trait ontologies) at the beginning of "Methods" section.

\section{Declarations}

Ethics approval and consent to participate Not applicable.

\section{Consent for publication}

Not applicable.

\section{Competing interests}

The author declares that he has no competing interests. 
Received: 21 December 2020 Accepted: 1 June 2021

Published online: 21 August 2021

\section{References}

1. Clayton JA, Collins FS. Policy: NIH to balance sex in cell and animal studies. Nature. 2014,509:282-3.

2. Sandberg K, Umans JG, Georgetown Consensus Conference Work Group. Recommendations concerning the new US National Institutes of Health initiative to balance the sex of cells and animals in preclinical research. FASEB J. 2015;29:1646-52.

3. Beery AK, Zucker I. Sex bias in neuroscience and biomedical research. Neurosci Biobehav Rev. 2011;35:565-72.

4. Prendergast BJ, Onishi KG, Zucker I. Female mice liberated for inclusion in neuroscience and biomedical research. Neurosci Biobehav Rev. 2014:40:1-5.
5. Itoh Y, Arnold AP. Are females more variable than males in gene expression? Meta-analysis of microarray datasets. Biol Sex Differ. 2015;6:18.

6. Varholick JA, Bailoo JD, Palme R, Wurbel H. Phenotypic variability between social dominance ranks in laboratory mice. Sci Rep. 2018;8:6593.

7. Mogil JS, Chanda ML. The case for the inclusion of female subjects in basic science studies of pain. Pain. 2005;117:1-5.

8. Bind RH, Minney SM, Rosenfeld S, Hallock RM. The role of pheromonal responses in rodent behavior: future directions for the development of laboratory protocols. J Am Assoc Lab Anim Sci. 2013;52:124-9.

\section{Publisher's Note}

Springer Nature remains neutral with regard to jurisdictional claims in published maps and institutional affiliations.
Ready to submit your research? Choose BMC and benefit from:

- fast, convenient online submission

- thorough peer review by experienced researchers in your field

- rapid publication on acceptance

- support for research data, including large and complex data types

- gold Open Access which fosters wider collaboration and increased citations

- maximum visibility for your research: over 100M website views per year

At BMC, research is always in progress.

Learn more biomedcentral.com/submissions 